\title{
Association of combined PD-L1 expression and tumour-infiltrating lymphocyte features with survival and treatment outcomes in patients with metastatic melanoma
}

\author{
C. Bence, ${ }^{1, \dagger}$ V. Hofman,,${ }^{1,2,3, \dagger}$ E. Chamorey, ${ }^{4}$ E. Long-Mira, ${ }^{1,2,3}$ S. Lassalle,,${ }^{1,2,3}$ A.F. Albertini, ${ }^{5}$ I. Liolios, ${ }^{6}$ \\ K. Zahaf, ${ }^{1}$ A. Picard, ${ }^{7}$ H. Montaudié, ${ }^{7}$ J.P. Lacour, ${ }^{7}$ (iD T. Passeron, ${ }^{7}$ (iD A.A. Andea, ${ }^{8}$ M. Ilie, ${ }^{12,3, *}$ iD, \\ P. Hofman ${ }^{1,2,3, *}$ \\ 'Laboratory of Clinical and Experimental Pathology, Pasteur Hospital, Université Côte d'Azur, University Hospital Federation \\ OncoAge, Nice, France \\ ${ }^{2}$ CNRS, INSERM, Institute of Research on Cancer and Ageing of Nice (IRCAN), Université Côte d'Azur, University Hospital Federation \\ OncoAge, Nice, France \\ ${ }^{3}$ Hospital-Related Biobank (BB-0033-00025), Pasteur Hospital, Université Côte d’Azur, University Hospital Federation OncoAge, \\ Nice, France \\ ${ }^{4}$ Biostatistics Unit, Antoine Lacassagne Comprehensive Cancer Center, Nice, France \\ ${ }^{5}$ Medipath Laboratory, Mougins, France \\ ${ }^{6}$ DIAG Laboratory, Nice, France \\ ${ }^{7}$ Department of Dermatology, Archet Hospital, Université Côte d'Azur, Nice, France \\ ${ }^{8}$ Department of Pathology, Michigan Medicine, University of Michigan, Ann Arbor, MI, USA \\ *Correspondence: M. llie and P. Hofman. E-mails: ilie.m@chu-nice.fr (MI) and hofman.p@chu-nice.fr (PH)
}

\begin{abstract}
Background Recent advances obtained with immune checkpoint inhibitors (ICls) targeting the programmed cell death-1 (PD-1) protein have significantly improved the outcome of patients with metastatic melanoma. The PD-L1 expression in tumour cells as detected by immunohistochemistry is a predictive biomarker in some solid tumours, but appears insufficient as prognostic or predictive factor of response to ICls in metastatic melanomas.

Objectives We investigated whether the presence and the features of pretreatment CD8 ${ }^{+}$tumour-infiltrating $\mathrm{T}$ lymphocytes (TILs) could be a complementary prognostic or predictive biomarker in patients with metastatic melanoma.

Methods In this retrospective study, we evaluated the association of PD-L1 expression $\geq 5 \%$ of tumour cells combined with TIL features (CD8, CD28, Ki67) with the overall survival (OS) among 51 patients treated with ICls and 54 patients treated with other treatment options (non-ICls).

Results PD-L1 positivity was observed in $33 \%$ and $39 \%$ of primary melanomas and matched metastases, respectively, with, however, poor concordance between the primary and the matched metastatic site $(\kappa=0.283)$. No significant association was noted between PD-L1 expression and CD8 ${ }^{+}$TIL profile analysed as single markers and OS or response to immunotherapy. Instead, their combined analysis in primary melanoma samples showed that the PD-L1-/CD8 ${ }^{+}$status was significantly associated with prolonged OS in the whole population $(P=0.04)$ and in the subgroup treated with non-ICls $(P=0.009)$. Conversely, the PD-L1+/CD8 ${ }^{+}$status was a good prognostic factor in patients treated with ICls $(P=0.022)$, whereas was significantly associated with poor prognosis in patients treated with non-ICls $(P=0.014)$. While the expression of CD28 was not related to outcome, the Ki67 expression was significantly associated with poor OS in the subgroup CD8 ${ }^{+}$TIL+/PD-L1- $(P=0.02)$.

Conclusions The pretreatment combination of PD-L1 expression with the level of CD8 ${ }^{+}$TILs could better assess OS and predict therapeutic response of patients with metastatic melanoma treated by either immunotherapy or other treatment regimens.
\end{abstract}

Received: 20 April 2019; Accepted: 20 September 2019

\section{Conflicts of interest}

M. Ilié has received honoraria for travel support and consulting/advisory roles for AstraZeneca, Bristol-Myers Squibb, Roche, Boehringer-Ingelheim and Merck \& Co. outside the submitted work. P. Hofman has received honoraria for travel support and consulting/advisory roles for AstraZeneca, Roche, Bristol-Myers Squibb, Novartis, Pfizer, MSD, Qiagen, Thermo Fisher, Biocartis and Merck \& Co. outside the submitted work.

†These authors contributed equally to this work. 


\section{Introduction}

Malignant melanoma is among the types of cancer whose incidence and mortality significantly increased in the last decades. A total of 232000 new cases are diagnosed, and more than 55000 patients die from a metastatic melanoma each year across the world. ${ }^{1}$ Metastatic melanoma represents a highly aggressive form of skin cancer, with an overall 5-year survival rate of $<20 \%$ and a median survival time of approximately 9 months for stage IV disease. $^{2}$

The advent of the immunotherapy strategies, in particular the immune checkpoint inhibitors (ICIs) targeting programmed death-1 (PD-1) and its ligand PD-L1, represented a true paradigm shift with an impressive 58\% increase in the 3-year median survival. $^{3-5}$ Following the first phase I study evaluating nivolumab, ${ }^{5}$ several clinical trials have investigated the use of immunohistochemically (IHC) expression of PD-L1 in tumour cells as a biomarker to predict response to anti-PD-1/PD-L1 ICIs. PD-L1 expression in tumour cells was found to predict a good response to immunotherapy; however, a significant clinical benefit was also observed in patients whose tumour did not express PD-L1. ${ }^{6,7}$ Durable response to ICIs is limited to a subset of melanoma patients, while $40 \%$ of patients do not respond to anti-PD-1 inhibitors in monotherapy. In most clinical trials, the expression of PD-L1 IHC alone did not allow optimal selection of responding patients. ${ }^{4}$ This biomarker currently appears insufficient to predict a therapeutic response to ICIs in patients with metastatic melanoma, paving the way for further research to optimize predictive tests.

Moreover, the prognostic value of the PD-L1 expression in patients with metastatic melanoma remains controversial, reportedly being associated with either poor or good prognosis according to various studies. ${ }^{8-11}$ Therefore, PD-L1 expression does not appear to be a reliable prognostic biomarker for routine practice.

While PD-L1 alone is currently inadequate, as prognostic and predictive marker in metastatic melanoma, other potential biomarkers are currently emerging. The presence of intratumour $\mathrm{CD}^{+}$tumour-infiltrating lymphocytes (CD8 $\left.{ }^{+} \mathrm{TIL}\right)$ could prove to be an important prognostic and predictive marker, particularly in association with the expression of PD-L1 in tumour cells. ${ }^{11}$ Some studies have shown the importance of the microenvironment including the analysis of CD8 ${ }^{+} \mathrm{TIL}$ and the potential correlation with a good response to anti-PD-1 inhibitors in some solid tumours, including metastatic melanoma. ${ }^{7,11-13}$

The classification of tumours into four groups, based on the presence or absence of CD8 ${ }^{+}$TILs and the expression of PD-L1 in tumour cells, has recently been proposed to predict the response to immunotherapy. ${ }^{14}$ However, studies evaluating the impact of such classification for the stratification of melanoma patients treated with immunotherapy are limited. ${ }^{15,16}$

Recent studies demonstrated that PD-1 suppresses T-cell function primarily by inactivating CD28 signalling, while the rescue of exhausted $\mathrm{CD}^{+}$TILs by $\mathrm{PD}-1$ targeted therapies is CD28-dependent, suggesting that T-cell costimulatory receptor CD28 could be a primary target for PD-1-mediated inhibition. $^{17,18}$

The objective of our study was to correlate the expression of PD-L1 in tumour cells, combined with the quantification of CD8 ${ }^{+}$TILs and their activation status (CD28, Ki67), to overall survival (OS) and response to treatment in order to determine whether this combination could be a more effective prognostic and predictive biomarker than IHC PD-L1 alone.

\section{Patients and methods}

\section{Study population}

This retrospective cohort included patients with primary cutaneous metastatic malignant melanoma (stage III/IV) diagnosed between July 2013 and February 2017 and treated at the Department of Dermatology, University of Nice, Archet 2 Hospital (Nice, France). The formalin-fixed paraffin-embedded (FFPE) tumour samples were retrieved from different laboratories: Laboratory of Clinical and Experimental Pathology (Pasteur Hospital, Nice, France), Medipath (Mougins, France), DIAG (Nice, France) and CAP (Nice, France). The availability of histological material from the primary tumour and metastasis was a required criterion to include a case in the study.

A total of 202 patients with metastatic melanoma were initially included in the study. Out of these, 97 (48\%) were excluded for various reasons (primary melanoma not available, small size of the sample, regressed primary melanoma, mucosal melanoma) leaving 105 patients in which the primary tumour and the first metastasis were available. Out of 105 patients, 91 presented with regional metastases (35 in transit and 56 lymph node metastases) and 14 with distant metastases (eight lung, and six subcutaneous).

The LDH levels at baseline were measured before initiation of the systemic treatment. The assay was performed in 76 patients.

Two groups of patients were distinguished for this study: a group of 51 patients $(48.5 \%)$ who received at least one treatment of immunotherapy (anti-PD-1 inhibitors - pembrolizumab/ nivolumab and/or anti-CTLA4) and a group of 54 patients (51.5\%) who have not had immunotherapy treatment, albeit some had other treatments (chemotherapy or targeted therapies 
with anti-BRAF and anti-MEK agents). Among the 51 patients of the group treated with immunotherapy, 29 (57\%) have had exclusively immunotherapy while $22(43 \%)$ have received an immunotherapy treatment before or after having other treatments (chemotherapy or targeted therapies).

All tumour specimens were used with the informed consent from the patients (Hospital-Integrated Biobank BB-0033-00025, Pasteur Hospital, Nice, France).

\section{Histology analysis}

Specimens were fixed in $10 \%$ formalin, embedded in paraffin and $4-\mu \mathrm{m}$-thick serial tissue sections obtained and stained with haematoxylin and eosin ( $\mathrm{H} \& \mathrm{E})$ for histologic evaluation. The H\&E-stained sections were independently assessed by two pathologists ( $\mathrm{CBE}$ and $\mathrm{VH}$ ), and the histopathological data including histologic subtype, Breslow thickness, ulceration and TNM stage were recorded. The primary malignant melanomas were reclassified with the 8th edition of the AJCC (American Joint Committee Cancer) tumour, node and metastasis classification. The density of the inflammatory infiltrate (none, mild, moderate, marked) and its location were assessed to determine the presence or the absence of TILs.

\section{Immunohistochemical analysis}

Formalin-fixed paraffin-embedded freshly cut serial 4 - $\mu$ m-thick tissue sections, mounted on positively charged slides, were stained for PD-L1 with the anti-human PD-L1 rabbit monoclonal antibody, clone 28-8 (kit PD-L1 PharmDx; Dako, Carpinteria, CA, USA). PD-L1 IHC was performed using a Autostainer Link 48 Dako-automated staining instrument, according to the manufacturer's recommendations. Staining was performed within two steps: a pre-pretreatment phase (PT Link Dako and EnVision FLEX Target Retrieval low pH solution) during $53 \mathrm{~min}$ and then the tissue sections were incubated with PD-L1 antibody during $30 \mathrm{~min}$ with the other components of the PD-L1 28-8 pharmDx kit, followed with a wash buffer (EnVision FLEX, Dako) and a 7-min counterstained with haematoxylin (EnVision FLEX, Dako).

In addition, CD8, CD28 and Ki67 IHC assays were performed using a BenchMark ULTRA-automated staining instrument (Ventana Medical Systems, Tucson, AZ, USA). FFPE freshly cut serial tissue sections were stained with a rabbit monoclonal antiCD8 antibody (clone SP57; Ventana), a rabbit polyclonal antiCD28 antibody (SIGMA Life Science, St. Louis, MO, USA) and a rabbit monoclonal anti-Ki67 antibody (clone 30-9; Ventana).

Each IHC run contained a positive control (tonsil) and a negative $\mathrm{Ab}$ control (buffer, no primary $\mathrm{Ab}$ ).

\section{Staining evaluation}

The IHC staining was independently assessed by two pathologists (CBE and $\mathrm{VH})$. When a discrepancy between the pathologists was noted, the slides were jointly reviewed on a multihead microscope with a third pathologist (MI or PH) to obtain a consensus.

PD-L1 staining was assessed on tumour cells and was considered positive if at least $5 \%$ of the tumour cells exhibited membranous PD-L1 staining of any intensity in a tissue section containing at least 100 cells that could be evaluated, as previously described in clinical trials using the corresponding antiPD1/PD-L1 inhibitors. ${ }^{4,19,20}$

Assessment of $\mathrm{CD}^{+}$TILs was performed by using Clark's modified grading system, as recommended by the International Immuno-Oncology Biomarkers Working Group. ${ }^{21,22}$ This grading system is based on the density (absent/mild/moderate/ marked, based on H\&E staining, score 0-3) and distribution (absent/focal/multifocal/diffuse, score 0-3) of TILs expressing CD8 at a threshold of $10 \% .^{21,22}$ TILs were defined as lymphocytes infiltrating and disrupting tumour nests and/or in direct contact with tumour cells. The possible combinations were collapsed into four TIL grades as follows: grade $0=$ absent; grade $1=$ mild or moderate focal infiltrate, or mild multifocal infiltrate; grade 2 = marked focal, moderate or marked multifocal or mild diffuse infiltrate; grade $3=$ moderate or marked diffuse infiltrate. Intense infiltrate referred to a strong heavy lymphocytic infiltrate of a density equivalent to that seen in a lymph node with metastasis. ${ }^{23}$ Intratumoral TILs were defined as lymphocytes infiltrating and disrupting tumour nests and/or in direct contact with the invasive tumour area as observed by haematoxylin and eosin staining. Peritumoral TILs were defined as lymphocytes located at distance from the tumour area, perivascular and inside the stromal fibrosis. The grades 1-3 TILs expressing CD8 at a threshold of $10 \%$ defined the CD $8{ }^{+}$TIL-positive group.

Primary tumours and the matched metastases were classified in four groups on the basis of their PD-L1 IHC status and presence (grade 1-3) or absence of $\mathrm{CD} 8^{+}$TILs: (i) $\mathrm{CD} 8^{+} \mathrm{TIL}+/ \mathrm{PD}-$ $\mathrm{L} 1+$; (ii) $\mathrm{CD} 8{ }^{+} \mathrm{TIL}-/ \mathrm{PD}-\mathrm{L} 1-$; (iii) $\mathrm{CD} 8^{+} \mathrm{TIL}+/ \mathrm{PD}-\mathrm{L} 1-$; and (iv) CD8 ${ }^{+}$TIL-/PD-L1+. In order to better define the activated $\mathrm{T}$ lymphocytes, CD28 and Ki67 were analysed only in the CD8 ${ }^{+}$TIL-positive group $(n=64)$.

CD28 staining was assessed considering the percentage of lymphocytes staining for CD28 compared to the lymphocytes staining for CD8. Similarly, the assessment of Ki67 was performed considering the percentage of lymphocytes stained for Ki67 compared to the lymphocytes staining for CD8.

\section{BRAF molecular analysis}

The BRAF mutational status was determined on tumour DNA isolated from FFPE tissue samples of melanoma metastases using the QIAamp DNA FFPE tissue kit (Qiagen, Hilden, Germany), according to the manufacturer's instructions. Pyrosequencing of BRAF exon 15 using the Therascreen $B R A F$ Pyro Kit (Qiagen) was performed as previously described. $^{24}$ 


\section{Statistical analysis}

All statistical analyses were performed at alpha risk $=5 \%$ under bilateral assumption using R.3.2.3 software (https://cran.r-projec t.org/bin/windows/base/old/3.2.3/) on Windows. Qualitative data were presented as absolute frequencies, percentages, $95 \%$ confidence intervals, missing data percentages. These data were compared using the chi-square test or the Fisher test in case of non-compliance with chi-square application conditions. Quantitative data were described by medians, extremes, means, standard deviations and percentages of missing data. These data were compared using Student's $t$-test or the Mann-Whitney test in case of non-compliance with the Student test conditions. Overall survival (OS) since primary was defined as the interval between the date of diagnosis of the primary tumour and the date of death of the patient or of the last follow-up. OS since the first metastasis was defined as the interval between the date of diagnosis of the first metastasis and the date of death of the patient or of the last follow-up. Patients lost to follow-up were censored on the date of last contact. These data were described by survival rates, survival medians and $95 \%$ confidence intervals. The survival curves were compared by the log-rank test. KaplanMeier survival curves were determined to assess the prognostic significance of PD-L1 expression and CD $8^{+}$TILs (grades $1-3$ ) on OS. The cut-off predicting OS for CD28 expression level and Ki67 index was evaluated graphically by using inflection points of the smoothing spline curve fit for both analytes and confirmed by using statistical rules assessed using $\mathrm{R}$ function 'bestcut2' for survival data model. Thus, the cut-off was defined at 20 for both analytes. Multivariate analyses were performed using Cox regression models with corresponding adjusted hazard ratio (HR) calculations. For the whole study, maximum patient follow-up was limited to 60 months. $P$-values $<0.05$ indicated statistical significance.

\section{Results}

\section{PD-L1 expression patterns}

The main clinical and histomolecular characteristics of this cohort are shown in Table 1 . The PD-L1 expression $\geq 5 \%$ tumour cells was observed in 35 out of 105 (33.3\%) primary melanomas (range, 5-50\%) and in 41 out of 105 (39\%) first metastasis of the matched primary melanoma (range, 5-100\%). There was a poor intrapatient concordance between PD-L1 expression status of the primary melanoma and the first metastatic site ( $\kappa=0.283 ; 95 \%$ CI, 0.072-0.494).

PD-L1 expression was membranous, essentially heterogeneous and focal, most often at the periphery of the tumour, located at the tumour invasion front (Fig. 1). The mononuclear cells of the tumour microenvironment were mostly lymphocytes with occasional plasma cells or macrophages. This infiltrate was most often located at the border of the tumour areas, as aggregates, and/or more rarely intratumoral as isolated cells. The topography and density of this infiltrate was clearly visible on the IHC CD8 staining (Fig. 2).

\section{Correlations with the clinicopathological characteristics}

There was a significant association between the PD-L1 expression status ( $\geq 5 \%$ tumour cells) and the presence of CD8 ${ }^{+}$TILs $(P=0.008$, Table 2$)$.

More than half of primary melanomas were infiltrated with CD8 ${ }^{+}$TILs (64/105; 60.9\%; grades $1-3$ ). The classification into four subgroups based on the PD-L1 status and the presence or absence of $\mathrm{CD}^{+}$TILs (grades 1-3) ${ }^{14}$ showed that the subgroups PD-L1-/TILs+ (34\%) and PD-L1-/TILs- (32\%) were the most represented, followed by the subgroup PD-L1+/TILs+ (27\%), while the subgroup PD-L1+/TILs- (7\%) was poorly represented (Table 2, Fig. 3).

In the metastatic lesions, $\mathrm{CD} 8^{+} \mathrm{TIL}-$ positive status was observed in $46.6 \%(49 / 105)$ of cases and the distribution of groups showed a predominance of the PD-L1-/TIL group (45\%) followed by the PD-L1+/TILs+ group (30\%) and a significant decrease in the PD-L1-/TILs+ infiltrates (16\%). The PD-L1+/ TILs- group was poorly represented $(9 \% ; P<0.001$, Table 2$)$.

There was no significant correlation between the CD28 and Ki67 expression in the 64 primary melanomas $(P=0.70)$. Moreover, there was no significant association between the PD-L1 status and gender $(P=0.46)$, histological subtype $(P=0.23)$, ulceration $(P=0.37)$, AJCC-T subgrouping $(P=0.54)$ or stage $(P=0.19)$, BRAF status $(P=0.39)$ and LDH level at baseline $(P=0.15)$.

No significant difference was observed for all of these data in the two groups of patients receiving or not immunotherapy treatment, except for age. Patients treated with immunotherapy were slightly younger than patients not receiving immunotherapy (median, 60 years vs. 66 years, $P=0.031$ ).

\section{Overall survival analysis in the whole population}

The pTNM stage, high baseline LDH levels and the presence of ulceration were significantly associated with poor OS in our population $(P=0.044, P=0.043$ and $P=0.00068$, respectively). Conversely, independently adjusted PD-L1 expression and $\mathrm{CD} 8^{+}$TIL status were not significantly correlated with OS ( $P=0.50$ and $P=0.27$, respectively).

The OS analysis according to the $\mathrm{CD} 8^{+}$TILs/PD-L1 status showed that the PD-L1-/TILs+ subgroup compared to the other groups analysed together demonstrated better OS $(P=0.041$, Fig. $4 \mathrm{~b})$. The percentage of patients alive at 60 months was $72 \%(26 / 36)$ for the PD-L1-/TILs+ subgroup compared to $50 \%(35 / 69)$ for all patients in the other three groups combined.

Among the 64 primary melanomas tested, the CD28 expression was not a significant prognostic factor for OS, independently of the cut-off $(20, P=0.253$; and 70 , $P=0.343$ respectively; data not shown). Similarly, when the CD28 expression was adjusted according to the subgroups 
Table 1 Clinical and histomolecular characteristics of the metastatic melanoma cohorts treated by chemotherapy or immunotherapy

\begin{tabular}{|c|c|c|c|c|}
\hline Characteristics & $\begin{array}{l}\text { Immunotherapy-naive } \\
\text { patients }(n=54), \%\end{array}$ & $\begin{array}{l}\text { Patients treated by } \\
\text { immunotherapy } \\
(n=51), \%\end{array}$ & $\begin{array}{l}\text { Total } \\
(n=105), \%\end{array}$ & $P$-value* \\
\hline Gender & & & & 0.30 \\
\hline Female & $21(60.00)$ & $14(40.00)$ & 35 (33.33) & \\
\hline Male & $33(47.14)$ & $37(52.86)$ & $70(66.67)$ & \\
\hline \multicolumn{5}{|l|}{ Age (years) } \\
\hline Mean & 66.59 & 60.55 & 63.66 & 0.031 \\
\hline Range & 23-92 & $26-83$ & 23-92 & \\
\hline LDH baseline & & & & 0.99 \\
\hline Normal & $26(40.00 \%)$ & $39(60.00 \%)$ & 65 & \\
\hline High & $5(45.45 \%)$ & $6(54.55 \%)$ & 11 & \\
\hline Histological subtype & & & & 0.83 \\
\hline $\begin{array}{l}\text { Superficial spreading } \\
\text { melanoma }\end{array}$ & $30(53.57)$ & $26(46.43)$ & $56(53.33)$ & \\
\hline Nodular melanoma & $15(46.88)$ & $17(53.12)$ & $32(30.48)$ & \\
\hline $\begin{array}{l}\text { Acral lentiginous } \\
\text { melanoma }\end{array}$ & $3(50.00)$ & $3(50.00)$ & $6(5.71)$ & \\
\hline $\begin{array}{l}\text { Invasive lentigo } \\
\text { maligna melanoma }\end{array}$ & $3(75.00)$ & $1(25.00)$ & $4(3.81)$ & \\
\hline Not classified & $3(42.85)$ & $4(57.14)$ & $7(6.67)$ & \\
\hline Ulceration & & & & 0.77 \\
\hline Absent & $25(49.02)$ & $26(50.98)$ & $51(48.57)$ & \\
\hline Present & $29(53.70)$ & $25(46.30)$ & $54(51.43)$ & \\
\hline pT stage & & & & 0.84 \\
\hline T1 & $7(58.33)$ & $5(41.67)$ & $12(11.43)$ & \\
\hline T2 & $10(55.56)$ & $8(44.44)$ & $18(17.14)$ & \\
\hline T3 & $20(52.63)$ & $18(47.37)$ & $38(36.19)$ & \\
\hline $\mathrm{T} 4$ & 17 (45.95) & $20(54.05)$ & $37(35.24)$ & \\
\hline pN stage & & & & 0.58 \\
\hline No & 47 (52.22) & $43(47.78)$ & $90(85.71)$ & \\
\hline $\mathrm{N} 1 \mathrm{a}$ & $0(0.00)$ & $1(100.00)$ & $1(0.95)$ & \\
\hline N1b & $0(0.00)$ & $1(100.00)$ & $1(0.95)$ & \\
\hline N1c & $6(60.00)$ & $4(40.00)$ & $10(9.52)$ & \\
\hline N3 & 1 (33.33) & $2(66.67)$ & $3(2.86)$ & \\
\hline Stage at diagnosis & & & & 0.53 \\
\hline 1 & $13(61.90)$ & $8(38.10)$ & $21(20.00)$ & \\
\hline ॥ & $34(50.00)$ & $34(50.00)$ & $68(64.76)$ & \\
\hline III & $7(46.67)$ & $8(53.33)$ & $15(14.28)$ & \\
\hline IV & $0(0.00)$ & $1(100.00)$ & $1(0.95)$ & \\
\hline$B R A F$ status & & & & 0.071 \\
\hline Mutation & $25(62.50)$ & $15(37.50)$ & $40(38.10)$ & \\
\hline Wild-type & $29(44.61)$ & $36(55.38)$ & $65(61.90)$ & \\
\hline
\end{tabular}

*Chi-square test or Student's t-test was used to investigate difference between groups.

PD-L1+/TILs+ and PD-L1-/TILs+, no significant difference was observed, regardless of the cut-off $(P=0.06, P=0.936$, $P=0.297, P=0.319$; data not shown).

In contrast, the Ki67 expression analysed alone and in the PD-L1-/CD8 ${ }^{+}$TIL + subgroup was a significant prognostic factor for poor OS ( $P=0.02$; data not shown).

The multivariate analysis, adjusted according to age, histological type, presence of ulceration and pTNM stage, showed that the PD-L1-/TILs+ subgroup was a significant independent prognostic factor for better OS (HR, 0.41; 95\% CI, 0.17-0.98; $P=0.04)$.

Finally, the univariate OS analyses described for the primary melanomas were also performed according to the expression levels on the first metastasis, regardless of the metastatic site. The expression of PD-L1, the status CD8 ${ }^{+}$TIL and CD8 ${ }^{+}$TIL/PD-L1 subgroups were not significantly related to OS (data not shown). 

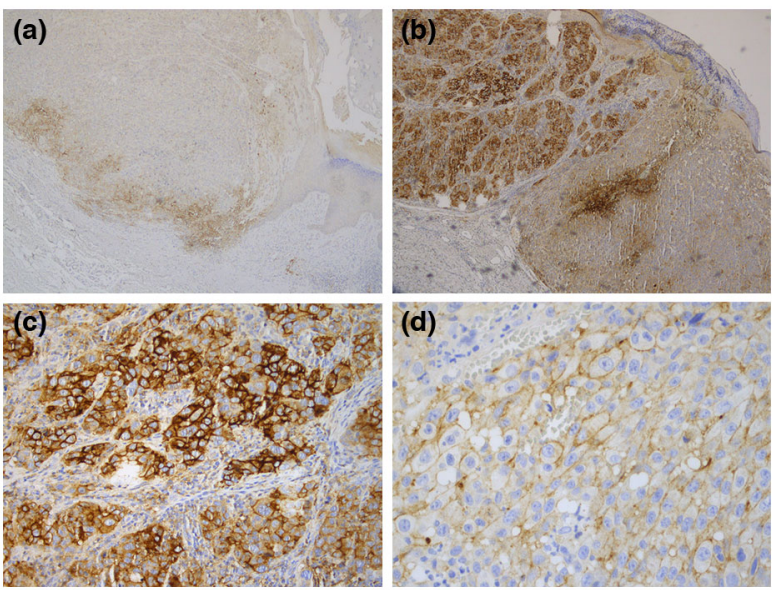

Figure 1 Various patterns of PD-L1 expression in melanocytic tumour cells. (a) PD-L1 expressed in $20 \%$ of tumour cells at the periphery of tumour areas (original magnification, $\times 100$ ). (b) Heterogeneous PD-L1 expression in $50 \%$ of tumour cells (original magnification, $\times 100$ ). (c) Strong membranous expression (original magnification, $\times 400$ ). (d) Low-to-moderate membranous expression (original magnification, $\times 400$ ).

\section{Overall survival analysis in primary melanoma not treated with immunotherapy}

In the population of patients who had never received immunotherapy, PD-L1 expression and $\mathrm{CD}^{+} \mathrm{TIL}$ status analysed alone were not significant prognostic factors $(P=0.11$ and $P=0.61$, respectively); data not shown). Conversely, the PD-L1/ $\mathrm{CD}^{+} \mathrm{TIL}$ status was significantly associated with favourable OS $(P=0.015$; Fig. 5a).

The PD-L1-/CD8 ${ }^{+}$TILs+ subgroup compared to the other three groups was significantly correlated with better OS $(P=0.009$; Fig. $5 b)$, with a percentage of patients alive at 60 months of $83 \%(15 / 18)$ vs. $47 \%$ for the other three groups combined (17/36). These results were confirmed in a multivariate analysis that showed that PD-L1-/TILs+ status was a good independent prognostic factor (HR, 0.05; 95\% CI, 0.007-0.36; $P=0.002)$.

In contrast, the PD-L1+/CD8 ${ }^{+} \mathrm{TILs}^{+}$status, compared to the other three groups, was a poor prognostic factor $(P=0.014$; Fig. 5 c $)$. The percentage of patients alive at 60 months was $37 \%(6 / 16)$ compared to $68 \%$ for the three combined groups (26/38). The PD-L1+/CD8 ${ }^{+}$TILs $^{+}$status was confirmed as a significant and independent prognostic factor associated with poor OS by multivariate analysis (HR, 6.7; 95\% CI, 1.63-27.93; $P=0.007)$.

\section{Overall survival analysis in primary melanoma treated with immunotherapy}

In the population of patients treated by immunotherapy, the PD-L1 expression and the CD8 ${ }^{+}$TIL status analysed alone were
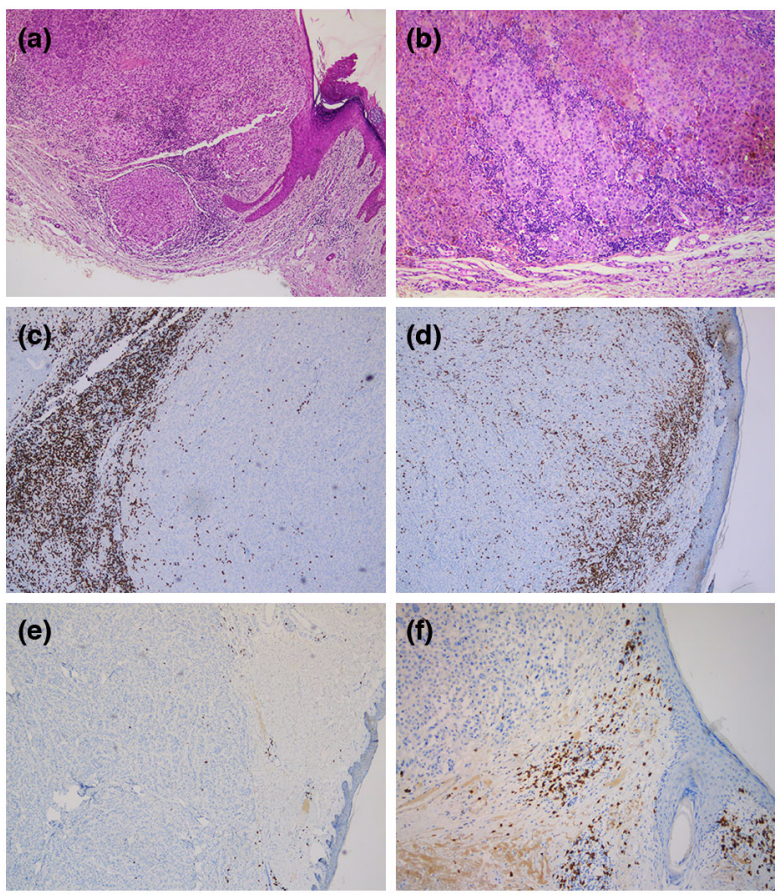

Figure 2 Distribution patterns of tumour-infiltrating lymphocytes (TILS). (a) HES slide (original magnification, $\times 100$ ). (b) HES slide (original magnification, $\times 200$ ). (c) Periphery excluded infiltrates of CD8 ${ }^{+}$TILs (original magnification, $\times 100$ ). (d) Periphery and intratumoral infiltrates of CD8 ${ }^{+}$TILs (original magnification, $\times 100$ ). (e) Immune-desert with the absence of CD8 ${ }^{+}$TILs (original magnification, $\times 100$ ). (f) Peritumoral CD8 ${ }^{+}$TIL infiltrates (original magnification, $\times 100)$.

not significant prognostic factors $(P=0.50$ and $P=0.30$, respectively; data not shown).

There was no significant difference in OS when analysing the PD-L1/CD8 ${ }^{+}$TIL groups individually $(P=0.63)$. Moreover, the

Table 2 PD-L1 expression and distribution of the CD8 ${ }^{+}$TILs and PD-L1/CD8 ${ }^{+}$TIL subgroups in primary melanomas and the paired metastases

\begin{tabular}{|c|c|c|c|}
\hline & Status & $\begin{array}{l}\text { Primary melanoma } \\
(n=105)\end{array}$ & $\begin{array}{l}\text { Metastases } \\
(n=105)\end{array}$ \\
\hline \multicolumn{4}{|l|}{ PD-L1 expression } \\
\hline & Negative & $70(67 \%)$ & $64(61 \%)$ \\
\hline & Positive & $35(33 \%)$ & $41(39 \%)$ \\
\hline \multicolumn{4}{|l|}{ CD8 ${ }^{+}$TILs } \\
\hline & Negative & $41(39 \%)$ & $56(53 \%)$ \\
\hline & Positive & $64(61 \%)$ & $49(47 \%)$ \\
\hline \multicolumn{4}{|c|}{ PD-L1/CD8+TIL subgroups } \\
\hline & PD-L1-/TILs- & $34(32 \%)$ & $47(45 \%)$ \\
\hline & PD-L1+/TILs- & $7(7 \%)$ & $9(9 \%)$ \\
\hline & PD-L1-/TILs+ & $36(34 \%)$ & $17(16 \%)$ \\
\hline & PD-L1+/TILs+ & $28(27 \%)$ & $32(30 \%)$ \\
\hline
\end{tabular}



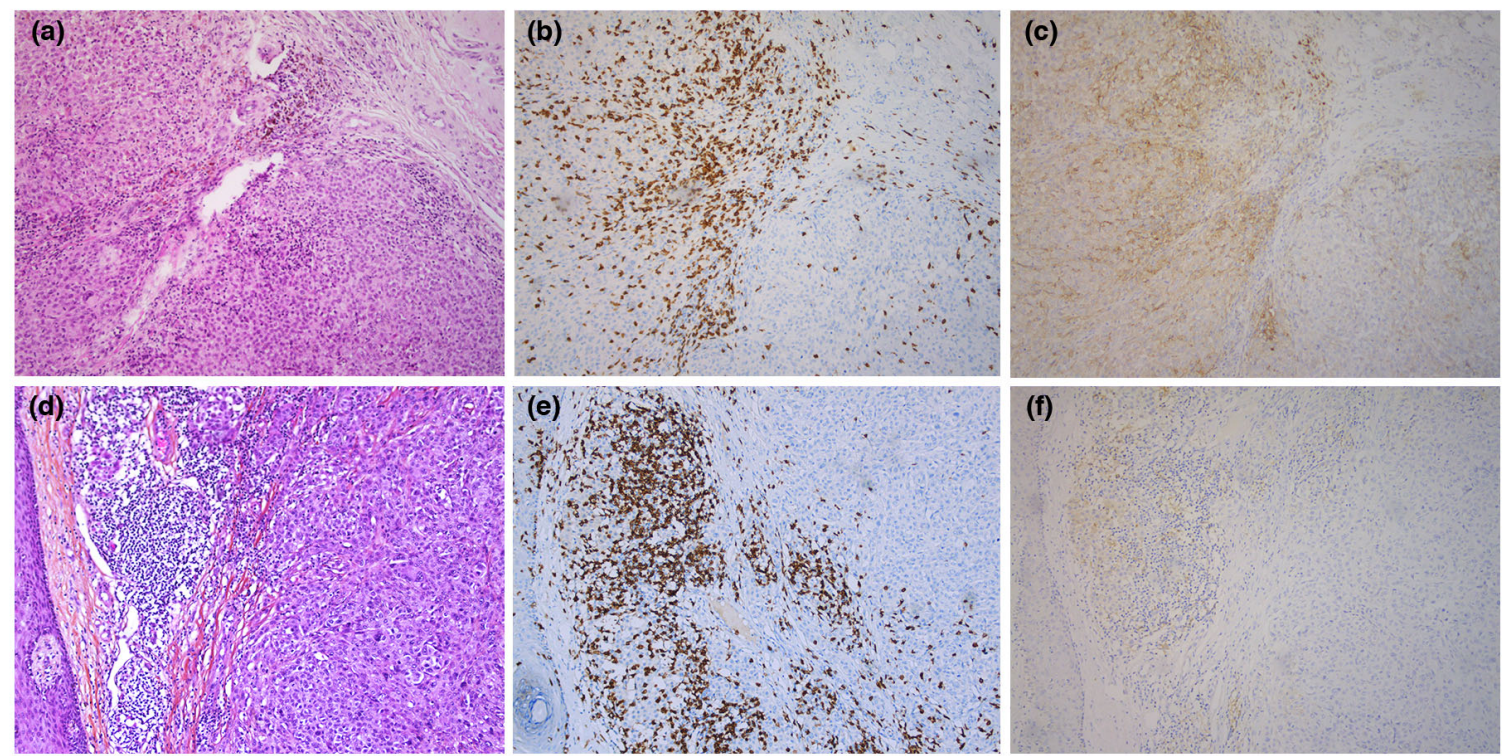

Figure 3 Patterns observed in the PD-L1+/CD8 ${ }^{+}$TILs+ and PD-L1-/CD8 ${ }^{+}$TILs + subgroups. Melanoma with inflammatory infiltrate (a, HES), demonstrating the presence of CD8 ${ }^{+}$TILs (b), associated with the expression of PD-L1 in tumour cells (c). Original magnification, $\times 100$. Melanoma with inflammatory infiltrate $(\mathrm{d}, \mathrm{HES})$, demonstrating the presence of CD8 ${ }^{+}$TILs (e), but no expression of PD-L1 in tumour cells (f). Original magnification, $\times 100$.

PD-L1-/CD8 ${ }^{+}$TIL status compared to the other three groups was not a significant prognostic factor for $\mathrm{OS}(P=0.86$; data not shown). As opposed to that, the PD-L1+/CD8 ${ }^{+}$TIL subgroup was correlated to better OS with an increased percentage of patients alive at 60 months compared to the other three groups $(66 \%, 8 /$ 12 of patients alive vs. $53 \%, 21 / 39 ; P=0.034$; Fig. $5 \mathrm{~d}$ ).

In the multivariate analysis, including age (HR, 2.277; 95\% CI, 0.356-8.02, $P=0.168$ ), histology (HR, 1.689; 95\% (a)

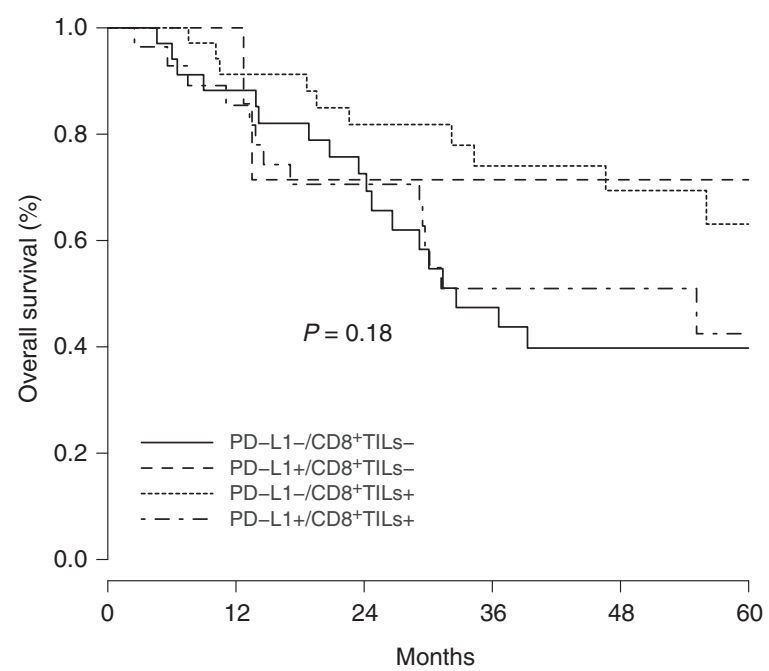

(b)

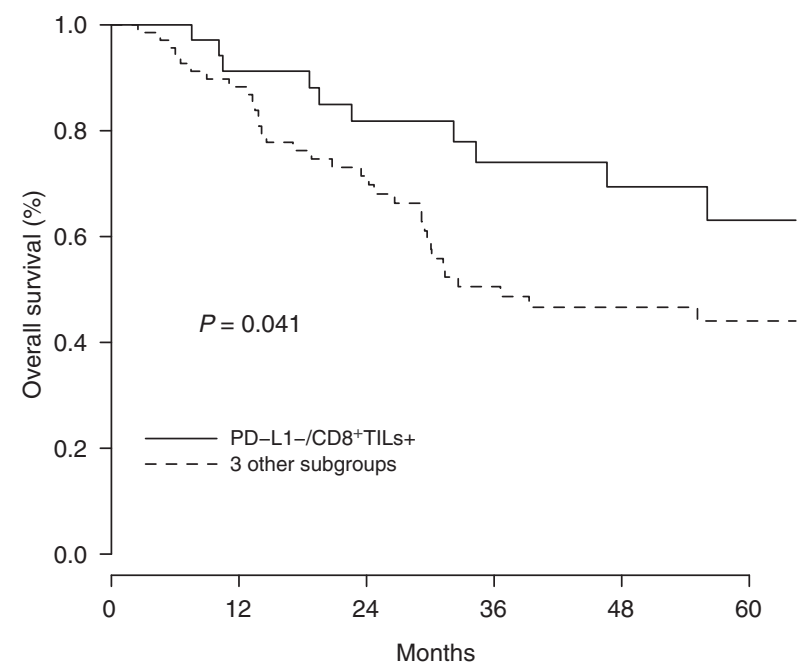

Figure 4 Kaplan-Meier overall survival curves according to PD-L1/CD8 ${ }^{+}$TIL status in the 105 primary melanomas. (a) Analysis of the four groups PD-L1/CD8 ${ }^{+}$TILs. (b) Analysis of the PD-L1-/CD8 ${ }^{+}$TIL group alone compared to the other three groups. The $P$-values were calculated using the log-rank test. 
(a)

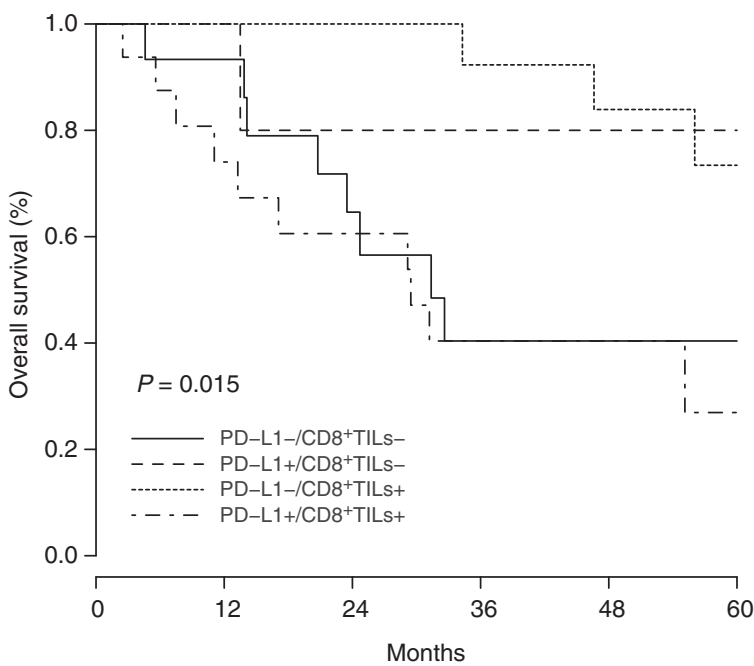

(c)

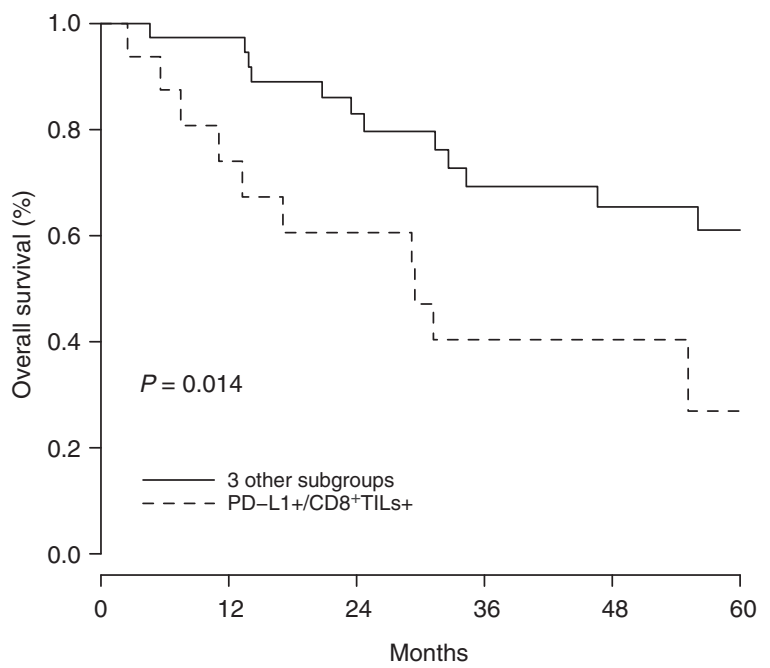

(b)

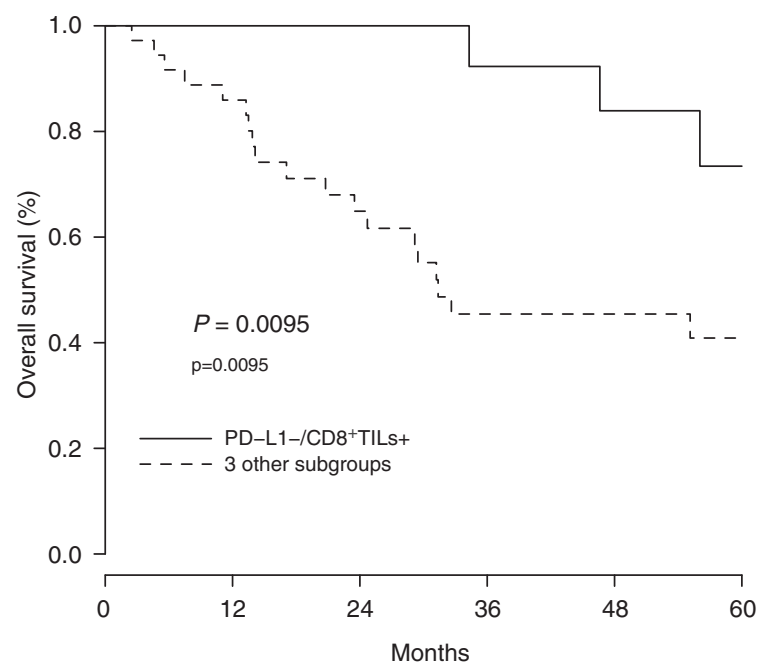

(d)

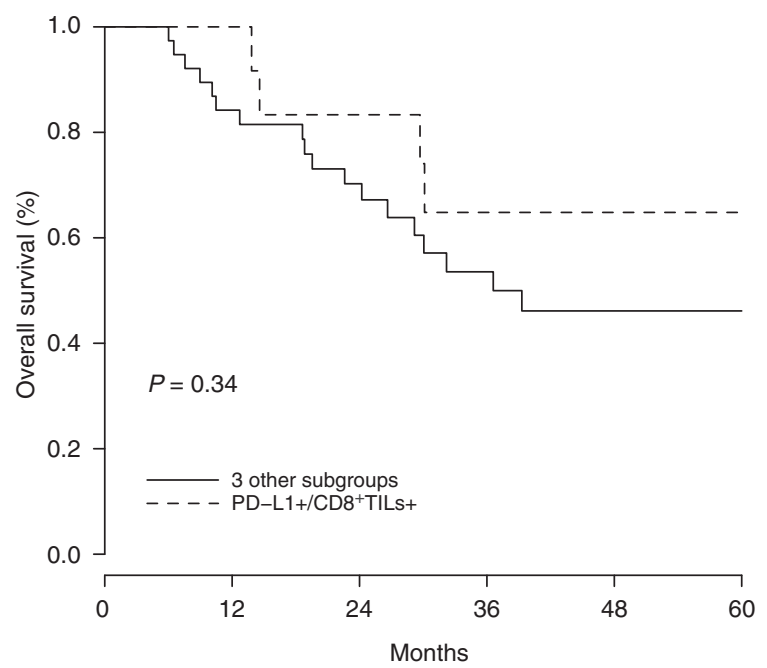

Figure 5 Kaplan-Meier overall survival curves according to PD-L1/CD8 ${ }^{+}$TIL status in the primary melanomas non-treated by immunotherapy $(n=54)$ or treated by immunotherapy $(n=51)$. (a) The four groups PD-L1/CD8 ${ }^{+}$TILs in patients non-treated by immunotherapy. (b) PD-L1-/CD8 ${ }^{+}$TILs+ group alone compared to the other three groups, in patients non-treated by immunotherapy. (c) PD-L1+/CD8 ${ }^{+}$TILs+ group alone compared to the other 3 groups, in patients non-treated by immunotherapy. (d) PD-L1+/CD8 ${ }^{+}$TILs + group alone compared to the other three groups, in patients treated by immunotherapy. The $P$-values were calculated using the log-rank test.

CI, 0.689-7.531; $P=0.501)$, stage $(\mathrm{HR}, 3.695 ; 95 \% \mathrm{CI}$, 1.213-11.257; $P=0.018$ ), ulceration (HR, 2.983; 95\% CI, 1.298-6.855; $P=0.008$ ) and baseline LDH levels (HR, 0.815; 95\% CI, 0.294-2.259; $P=0.687)$, the stage and PD-L1+/ TILs+ status were significant and independent prognostic factors associated with OS compared to the other groups (PD-L1+/TILs+ status; HR, 0.138; 95\% CI, 0.024-0.779; $P=0.022$ ).

\section{Discussion}

Treatment with ICIs in patients with metastatic melanoma demonstrates impressive response rates. However, although the benefit is restricted to approximately $40 \%$ of patients treated with anti-PD-1 therapy, there are no approved stratification strategies for immunotherapy in melanoma. ${ }^{25}$ Thus, there is an acute need for robust predictive biomarkers to guide the clinical decision-making. ${ }^{26}$ 
Melanoma is one of the tumours with the highest somatic mutation rates among solid tumours, which is thought to be related to their high immunogenicity (i.e. their ability to induce an adaptive immune response specifically directed against tumour antigens). ${ }^{27}$ In melanoma, the PD-L1 expression in tumour cells is in most cases adaptive, being rarely related to constitutive the activation of mitogen-activated protein kinase pathways. $^{7,28}$

In agreement with previous studies, we found that the adaptive PD-L1 response is constituted by focal expression in tumour cells, often at the periphery of the tumour, at the tumour invasion front, and close to an inflammatory CD8 ${ }^{+}$TIL. $^{15}$

The PD-L1 status is notoriously difficult to estimate due to the variability of methods used across studies but also related to the intrinsic intra- and inter-tumour heterogeneity.

The IHC tests used in the majority of clinical trials employed two different clones (e.g. 28-8 and 22C3), and the positivity thresholds vary from $1 \%$ to $5 \%$ of tumour cells. Moreover, in some studies the PD-L1-positive immune cells were also taken into account in evaluating the levels of expression and calculating the positivity threshold. ${ }^{25}$ In clinical trials, the most widely used positivity threshold is $\geq 5 \%$ of tumour cells when using the pharmDx 28-8 kit. $^{4,5,20,29}$

Second, there are significant variations in PD-L1 expression within the same tumour and between samples from different tumour sites in the same patient. ${ }^{30,31}$ Given the intratumour heterogeneity and in order to analyse the largest tumour area, we evaluated the expression of PD-L1 on whole tissue sections, which can better reflect the distribution and type of cells expressing PDL1 and also allows assessing the relationship between PD-L1 expression on tumour cells and on immune cells.

As a consequence of the adaptive immune resistance mechanism and the existence of immunogenic or non-immunogenic tumours, the infiltration of immune cells into the tumour, particularly $\mathrm{T}$ cells, associated with the expression of PD-L1 could be an important predictive biomarker for PD-1/PD-L1 checkpoint inhibitors. $^{32}$

In our study, the proportion of primary melanomas expressing PD-L1 on tumour cells was 33\%, similar to some previous reports, ${ }^{15}$ while other studies reported higher rates. One study observed PD-L1 positivity in $51 \%$ of cases, but with a positivity threshold of $1 \%,{ }^{30}$ whereas another study using a $5 \%$ threshold reported 53\% $\mathrm{PD}-\mathrm{L} 1$ - positive rate; however, the site of sampling, primary or metastatic, was not specified. ${ }^{7}$

Most studies that have investigated the predictive value of PD-L1 expression for the response to anti-PD-1/PD-L1 therapy in metastatic melanomas have not distinguished the primary or metastatic site. ${ }^{7,13,19}$ In our study, the PD-L1 expression was more frequent in metastases (39\% vs. 33\%). Our results are similar to a study by Taube et al. ${ }^{15}$ (43\% vs. $\left.35 \%\right)$, while conflicting results have been reported by Madore et al. ${ }^{30}$ (51\% for primary, $57 \%$ for regional metastases and $42 \%$ for distant metastases).
We did not find any significant association between PD-L1 expression on tumour cells of primary melanoma and OS, as previously shown. ${ }^{15,33}$ In melanoma, the prognostic significance of PD-L1 expression remains controversial, being associated with either a poor ${ }^{8-10}$ or a better prognosis. ${ }^{11}$

In our cohort, PD-L1 expression was not significantly associated with the response of PD-1/PD-L1 agents. Whereas the expression of PD-L1 by tumour cells may be predictive of a good response to immunotherapy, nevertheless, a significant clinical benefit can also be observed in most studies in the negative PD-L1 group, even if response rates remain lower. ${ }^{6,7,34}$ Overall, the variability of the methods used, the absence of a standardized positivity threshold, the spatio-temporal heterogeneity and the presence of an immunotherapy response in $\mathrm{PD}-\mathrm{L} 1-$ negative patients are such that $\mathrm{PD}-\mathrm{L} 1$ does not appear to be a good standalone biomarker for response to immunotherapy in melanoma.

The presence of intratumoral $\mathrm{CD}^{+}$TILs is frequently observed in melanomas. ${ }^{15}$ In our study, $60.9 \%$ of primary melanomas had a positive $\mathrm{CD} 8^{+}$TIL status with a lower rate in the metastatic samples, for any site combined (46.6\%).

In the whole population, we have not found a significant association between the presence of $\mathrm{CD} 8{ }^{+}$TILs in primary melanoma or metastases and OS, as previously observed in other series. ${ }^{32,35}$ Most studies have shown that the presence of a high density of TILs in primary melanomas was significantly associated with better outcome, ${ }^{36,37}$ suggesting that a functional lymphocyte infiltration is necessary for the effectiveness of PD-1/PD-L1 blockage. Moreover, the $\mathrm{CD} 8^{+} \mathrm{TIL}$ status, studied alone in our series, was not predictive of response, unlike previous reports. $^{13,34}$

However, the presence of $\mathrm{CD} 8^{+} \mathrm{TIL}$ infiltrate in the tumour could be an important clinical biomarker when combined to PD-L1 expression. Two studies suggested that the expression of PD-L1 should be interpreted taking into account the tumour microenvironment. The tumours were categorized into 4 different types of tumour microenvironment based on the presence or absence of TILs and PD-L1 expression, suggesting that this classification could be more effective to predict the response to immunotherapy. ${ }^{14,15}$

In our study, the association between the PD-L1 expression and the presence of CD8 ${ }^{+}$TILs was found in $26.6 \%$ of the primary melanomas, the majority groups being the CD ${ }^{+} \mathrm{TIL}-/$ PD-L1- and CD8 ${ }^{+}$TIL+/PD-L1- groups. PD-L1 expression without associated $\mathrm{CD} 8^{+}$TILs was rare.

These observations were different on metastatic samples. The $\mathrm{CD} 8^{+}$TIL-/PD-L1 - group became the majority while the $\mathrm{CD} 8{ }^{+} \mathrm{TIL}+/ \mathrm{PD}-\mathrm{L} 1-$ group was half as high as in primary melanomas. It is interesting to note that approximately the same distribution of these different groups was found by Taube et al., ${ }^{15}$ on both primary and metastatic melanomas. A review of the literature reports a different distribution showing a predominance 
of $\mathrm{CD}^{+}{ }^{+} \mathrm{TIL}+/ \mathrm{PD}-\mathrm{L} 1+(38 \%)$ and $\mathrm{CD}^{+}{ }^{\mathrm{TIL}}-/ \mathrm{PD}-\mathrm{L} 1-(41 \%)$ status but without distinction between primary and metastatic melanomas. ${ }^{14}$

Certain $\mathrm{CD}^{+}{ }^{+}$TIL/PD-L1 subgroups demonstrated prognostic and predictive value in the 105 primary melanomas, depending on the patient group studied, general population, patients treated or not by immunotherapy.

Thus, the group of patients with PD-L1-/CD8 ${ }^{+}$TILs+ status is a group with a good independent prognosis in the general population and even more significantly in the group of patients not treated with immunotherapy. Conversely, in the group of patients treated with immunotherapy, this profile was not a significant prognostic factor for OS. It would appear that patients with PD-L1-/CD8 ${ }^{+}$TILs + tumours are not good responders to immunotherapy. Indeed, immunotherapy does not seem to improve their survival.

Interestingly, our results suggest that for the choice of firstline treatment of mutated BRAF patients, the PD-L1-/ CD $8^{+}$TILs + subgroup could benefit from a combination of targeted therapy (anti-BRAF and anti-MEK). On the other hand, the PD-L1+/CD8 ${ }^{+}$TILs + subgroup, which is correlated with a good prognosis when treated with immunotherapy, could benefit from first-line immunotherapy, despite the mutated $B R A F$ status. There was no significant correlation between PD-L1 expression and BRAF mutation status, as previously showed in the literature. $^{38}$

Furthermore, we showed that the PD-L1+/CD8 ${ }^{+}$TILs + profile was an independent factor of poor prognosis, significantly observed in the group of patients not treated with immunotherapy, with only $37 \%$ of patients alive at 60 months. Conversely, in patients treated by immunotherapy, this profile was an independent factor associated with better outcome, with $66 \%$ of patients still alive at 60 months, as previously reported in cutaneous melanomas. ${ }^{13,39}$ In the whole study population and without distinguishing therapeutic management, these results were not significantly discriminated. The tendency of the PD-L1+/CD8 ${ }^{+}$TILs+ subgroup to have a poor prognosis appears to be masked in the general population by patients who have received immunotherapy and whose prognosis has been improved.

With regard to the PD-L1-/CD8 ${ }^{+}$TILs- profile, we could not show a prognostic or predictive significance for response to immunotherapy. However, the percentage of patients alive at 60 months in the general population and in the groups of patients treated or not with immunotherapy remained stable around $47 \%$. This suggests that the absence of CD8 ${ }^{+}$TILs associated with the absence of PD-L1 expression may be a poor prognostic factor, associated with the absence of response to immunotherapy.

Teng et al. ${ }^{14}$ suggest that, given the absence of pre-existing functional $\mathrm{T}$ lymphocytes, the PD-L1-/CD8 ${ }^{+}$TILs- profile would be associated with poor prognosis and patients would then likely not respond to anti-PD1 monotherapy, whereas the combination of anti-CTLA4 and anti-PD1 agents would be beneficial for these patients. Indeed, anti-CTLA4 would allow early activation of $\mathrm{T}$ cells that would be recruited into the tumour and induce adaptive expression of PD-L1, which would then be the target of anti-PD $1 .{ }^{14}$ The immunotherapy combinatorial strategies were not in the scope of our study; however, such strategies are of high importance for tumours that do not express PD-L1, and it would therefore be interesting to study the efficacy based on the four PD-L1/CD ${ }^{+}$TIL patterns. $^{29,40}$

Finally, the PD-L1+/CD8 ${ }^{+}$TILs - status was not found to be a prognostic nor predictive factor for a response to immunotherapy in any of our patient groups. However, this status, reflecting a constitutive expression of PD-L1, is very poorly represented in our series, as in the various series described in the literature. ${ }^{15}$ Finally, while the CD28 expression was not significantly associated with outcome, the Ki67 expression was significantly associated with poor survival in the PD-L1-/CD $8^{+}$TILs+, suggesting that the prognostic value may be driven by activated $\mathrm{CD} 8{ }^{+} \mathrm{T}$ cells in the absence of PD-L1 expression.

There are a number of limitations in our study. This is a single-institutional retrospective study with a modest sample size, even though all available cases at Nice University Hospital were collected at the time of the study. Although the hypothesis of this study is well-substantiated in the literature, an independent validation cohort would be optimal.

Another limitation is related to the heterogeneity of the 51 patients treated with immunotherapy. Although the majority of patients received anti-PD-1 monotherapy, few patients had two different lines of immunotherapy (anti-CTLA4 followed by antiPD-1) and more rarely other types of associated treatments (chemotherapy or targeted therapy) during the follow-up.

In summary, our study shows that PD-L1 expression alone is not a robust prognostic factor in patients with metastatic malignant melanoma, whether this status is assessed on the primary tumour or on the matched metastasis. In exchange, the additional assessment of the CD8 ${ }^{+}$TIL infiltrates could better assess the OS of subgroups of patients and predict the therapeutic response of patients with metastatic melanoma treated by either immunotherapy or other treatment regimens. Moreover, this IHC assay can be easily applied to current conventional routine testing and may offer valuable clinical information when considering different treatment options in the absence of established methods for patient stratification.

\section{References}

1 Bray F, Ferlay J, Soerjomataram I et al. Global cancer statistics 2018: GLOBOCAN estimates of incidence and mortality worldwide for 36 cancers in 185 countries. CA Cancer J Clin 2018; 68: 394-424.

2 Gershenwald JE, Scolyer RA, Hess KR et al. Melanoma staging: evidencebased changes in the American Joint Committee on Cancer eighth edition cancer staging manual. CA Cancer J Clin 2017; 67: 472-492. 
3 Topalian SL, Hodi FS, Brahmer JR et al. Safety, activity, and immune correlates of anti-PD-1 antibody in cancer. N Engl J Med 2012; 366: 2443-2454.

4 Robert C, Long GV, Brady B et al. Nivolumab in previously untreated melanoma without BRAF mutation. N Engl J Med 2015; 372: 320-330.

5 Wolchok JD, Chiarion-Sileni V, Gonzalez R et al. Overall survival with combined nivolumab and ipilimumab in advanced melanoma. $N$ Engl J Med 2017; 377: 1345-1356.

6 Gandini S, Massi D, Mandala M. PD-L1 expression in cancer patients receiving anti $\mathrm{PD}-1 / \mathrm{PD}-\mathrm{L} 1$ antibodies: a systematic review and meta-analysis. Crit Rev Oncol Hematol 2016; 100: 88-98.

7 Taube JM, Klein A, Brahmer JR et al. Association of PD-1, PD-1 ligands, and other features of the tumor immune microenvironment with response to anti-PD-1 therapy. Clin Cancer Res 2014; 20: 5064 5074.

8 Massi D, Brusa D, Merelli B et al. PD-L1 marks a subset of melanomas with a shorter overall survival and distinct genetic and morphological characteristics. Ann Oncol 2014; 25: 2433-2442.

9 Wang Q, Liu F, Liu L. Prognostic significance of PD-L1 in solid tumor: an updated meta-analysis. Medicine (Baltimore) 2017; 96: e6369.

10 Hino R, Kabashima K, Kato Y et al. Tumor cell expression of programmed cell death-1 ligand 1 is a prognostic factor for malignant melanoma. Cancer 2010; 116: 1757-1766.

11 Kluger HM, Zito CR, Barr ML et al. Characterization of PD-L1 expression and associated T-cell infiltrates in metastatic melanoma samples from variable anatomic sites. Clin Cancer Res 2015; 21: 3052-3060.

12 Herbst RS, Soria JC, Kowanetz M et al. Predictive correlates of response to the anti-PD-L1 antibody MPDL3280A in cancer patients. Nature 2014 515: 563-567.

13 Tumeh PC, Harview CL, Yearley JH et al. PD-1 blockade induces responses by inhibiting adaptive immune resistance. Nature 2014; 515: 568-571.

14 Teng MW, Ngiow SF, Ribas A et al. Classifying cancers based on T-cell infiltration and PD-L1. Cancer Res 2015; 75: 2139-2145.

15 Taube JM, Anders RA, Young GD et al. Colocalization of inflammatory response with B7-h1 expression in human melanocytic lesions supports an adaptive resistance mechanism of immune escape. Sci Transl Med 2012; 4: 127ra37.

16 Madonna G, Ballesteros-Merino C, Feng Z et al. PD-L1 expression with immune-infiltrate evaluation and outcome prediction in melanoma patients treated with ipilimumab. Oncoimmunology 2018; 7: e1405206.

17 Hui E, Cheung J, Zhu J et al. T cell costimulatory receptor CD28 is a primary target for PD-1-mediated inhibition. Science 2017; 355: 1428-1433.

18 Kamphorst AO, Wieland A, Nasti T et al. Rescue of exhausted CD8 T cells by PD-1-targeted therapies is CD28-dependent. Science 2017; 355: 1423-1427.

19 Wolchok JD, Kluger H, Callahan MK et al. Nivolumab plus ipilimumab in advanced melanoma. N Engl J Med 2013; 369: 122-133.

20 Weber JS, D'Angelo SP, Minor D et al. Nivolumab versus chemotherapy in patients with advanced melanoma who progressed after anti-CTLA-4 treatment (CheckMate 037): a randomised, controlled, open-label, phase 3 trial. Lancet Oncol 2015; 16: 375-384.

21 Hendry S, Salgado R, Gevaert T et al. Assessing tumor-infiltrating lymphocytes in solid tumors: a practical review for pathologists and proposal for a standardized method from the International ImmunoOncology Biomarkers Working Group: part 2: TILs in melanoma, gastrointestinal tract carcinomas, non-small cell lung carcinoma and mesothelioma, endometrial and ovarian carcinomas, squamous cell carcinoma of the head and neck, genitourinary carcinomas, and primary brain tumors. Adv Anat Pathol 2017; 24: 311-335.
22 Azimi F, Scolyer RA, Rumcheva P et al. Tumor-infiltrating lymphocyte grade is an independent predictor of sentinel lymph node status and survival in patients with cutaneous melanoma. J Clin Oncol 2012; 30: 26782683.

23 Brambilla E, Le Teuff G,jdv Marguet S et al. Prognostic effect of tumor lymphocytic infiltration in resectable non-small-cell lung cancer. J Clin Oncol 2016; 34: 1223-1230.

24 Ilie MI, Lassalle S, Long-Mira E et al. Diagnostic value of immunohistochemistry for the detection of the BRAF(V600E) mutation in papillary thyroid carcinoma: comparative analysis with three DNA-based assays. Thyroid 2014; 24: 858-866.

25 Ribas A, Hamid O, Daud A et al. Association of pembrolizumab with tumor response and survival among patients with advanced melanoma. JAMA 2016; 315: 1600-1609.

26 Tray N, Weber JS, Adams S. Predictive biomarkers for checkpoint immunotherapy: current status and challenges for clinical application. Cancer Immunol Res 2018; 6: 1122-1128.

27 Lawrence MS, Stojanov P, Polak P et al. Mutational heterogeneity in cancer and the search for new cancer-associated genes. Nature 2013; 499: 214-218

28 Berry S, Taube JM. Innate vs. adaptive: PD-L1-mediated immune resistance by melanoma. Oncoimmunology 2015; 4: e1029704.

29 Larkin J, Chiarion-Sileni V, Gonzalez R et al. Combined nivolumab and ipilimumab or monotherapy in untreated melanoma. N Engl J Med 2015; 373: 23-34.

30 Madore J, Vilain RE, Menzies AM et al. PD-L1 expression in melanoma shows marked heterogeneity within and between patients: implications for anti-PD-1/PD-L1 clinical trials. Pigment Cell Melanoma Res 2015; 28: 245-253.

31 Yang S, Leone DA, Biswas A et al. Concordance of somatic mutation profiles (BRAF, NRAS, and TERT) and tumoral PD-L1 in matched primary cutaneous and metastatic melanoma samples. Hum Pathol 2018; 82: 206 214.

32 Wong PF, Wei W, Smithy JW et al. Multiplex quantitative analysis of tumor-infiltrating lymphocytes and immunotherapy outcome in metastatic melanoma. Clin Cancer Res 2019; 25: 2442-2449.

33 Steiniche T, Vestergaard Danielsen A, Wang Z et al. PD-L1 expression and survival among melanoma patients treated with standard immunotherapy or chemotherapy. J Eur Acad Dermatol Venereol 2017; 31: e319-e321.

34 Daud AI, Wolchok JD, Robert C et al. Programmed death-ligand 1 expression and response to the anti-programmed death 1 antibody pembrolizumab in melanoma. J Clin Oncol 2016; 34: 4102-4109.

35 Burton AL, Roach BA, Mays MP et al. Prognostic significance of tumor infiltrating lymphocytes in melanoma. Am Surg 2011; 77: 188-192.

36 Lee N, Zakka LR, Mihm MC Jr et al. Tumour-infiltrating lymphocytes in melanoma prognosis and cancer immunotherapy. Pathology 2016; 48: 177-187.

37 Clemente CG, Mihm MC Jr, Bufalino R et al. Prognostic value of tumor infiltrating lymphocytes in the vertical growth phase of primary cutaneous melanoma. Cancer 1996; 77: 1303-1310.

38 Rodic N, Anders RA, Eshleman JR et al. PD-L1 expression in melanocytic lesions does not correlate with the BRAF V600E mutation. Cancer Immunol Res 2015; 3: 110-115.

39 Frydenlund N, Leone D, Yang S et al. Tumoral PD-L1 expression in desmoplastic melanoma is associated with depth of invasion, tumor-infiltrating CD8 cytotoxic lymphocytes and the mixed cytomorphological variant. Mod Pathol 2017; 30: 357-369.

40 Chae YK, Arya A, Iams W et al. Current landscape and future of dual anti-CTLA4 and PD-1/PD-L1 blockade immunotherapy in cancer; lessons learned from clinical trials with melanoma and non-small cell lung cancer (NSCLC). J Immunother Cancer 2018; 6: 39. 